\title{
Validez de las Escalas de Braden y EVARUCI en Pacientes Hospitalizados en una Unidad de Cuidados Intensivos
}

\author{
Validity of the Braden and EVARUCI Scales in Hospitalized Patients in an Intensive Care Unit
}

Margarita María Zapata Rodríguez, M.Sc ${ }^{1}$, Carmen Lorena Murillo Panameño M.Sc ${ }^{2}$, Juan Carlos Millán Estupiñan Mg. Epidemiología. ${ }^{2}$

1. Docente Universidad Libre, Cali - Colombia.

2. Docente Universidad Santiago de Cali. Colombia

\section{Resumen}

Con el fin de establecer diferencias entre las escalas de valoración de riesgo de desarrollo de ulceras por presión (Braden y EVARUCI) en pacientes críticos, como los que se encuentran hospitalizados en las unidades de cuidado intensivo. Se realizó la validez de ambas escalas determinando la sensibilidad, especificidad y valores predictivos. Para el estudio se seleccionaron consecutivamente pacientes mayores de edad, sin ulceras por presión en cualquier estadio, que ingresaron para ser hospitalizados con diagnóstico de cualquier patología. Se determinó que no hubo una verdadera diferencia entre la sensibilidad de ambas escalas, que la especificidad de la escala de EVARUCI fue mayor al igual que el valor predictivo positivo y no se evidenció diferencias significativas en el valor predictivo negativo. Se pudo concluir que la escala de EVARUCI es un instrumento predictor valido para la detección del riesgo de desarrollo de UPP ya que además valora aspectos, como nivel de consciencia, estado hemodinámico, soporte de oxígeno, movilidad, temperatura, saturación, entre otros.

Palabras Clave: Ulcera por presión (UPP) - unidad de cuidado intensivo - factores de riesgo - escalas de valoración - validez. (MESH)

\section{Abstract}

With the final purpose to establish difference between the titration scales of risk development pressure ulcers (Braden y EVARUCI) in critical patients, as those who are hospitalized in intensive care units. The validity of both scales was performed to determining the sensibility, the specificity and predictive values. For the study were selected consecutively patients of legal age, without pressure ulcers at any stage, who were admitted to be hospitalized with the diagnostic of any pathology. It was determined that there was not real difference between sensibility of both scales, that specificity of the scale of EVARUCI was higher like the positive predictive and evidence no significant differences in the negative predictive value. It was concluded that the scale of EVARUCI is the valid predictive instrument for the detection of the risk development for pressure ulcers, as besides valued aspects, like the level of consciousness, hemodynamic status, oxygen support, movility, tempeture, saturation, among others.

Keywords: Pressure ulcer - intensive care unit - risk factors - titration scale - validity.

\section{Introducción}

Una úlcera por presión es una lesión que se desarrolla en la piel y en los tejidos subyacentes debido a la presión continua, generalmente, sobre una prominencia ósea ${ }^{1}$.

La Organización Mundial de la Salud considera las úlceras por presión (UPP) como un indicador de la calidad asistencial, es decir un indicador de la calidad de los cuidados ofertados, tanto a los pacientes que las presentan como a los que corren riesgo de desarrollarlas ${ }^{2}$. Las úlceras por presión constituyen un importante problema porque repercuten en el nivel de salud y calidad de vida de quienes las presentan; en sus entornos, cuidadores y en el consumo de recursos del Sistema de Salud ${ }^{3}$. $\mathrm{Su}$ aparición también implica responsabilidad legal por mala práctica profesional, ya que se las considera un problema evitable en un $95 \%{ }^{4}$. La aparición de las úlceras por presión eleva la morbilidad y la mortalidad del paciente.

El primer paso para la prevención de las úlceras por presión es utilizar una escala de valoración del riesgo para desarrollarlas, ya que constituyen una herramienta de cribado, diseñada para ayudar al profesional asistencial a identificar

los pacientes que pueden desarrollar estas lesiones. A través de estas escalas se podrán planificar los cuidados necesarios adaptados a cada paciente para evitar la aparición de las úlceras.

Las Escalas de Valoración del Riesgo de Presentar Úlceras Por Presión (EVRUPP) deben tener un aspecto importante: La validez que es la demostración de que se está midiendo, aquello que se pretende medir, en este caso, el riesgo de presentar una úlcera por presión. Los indicadores de validez más utilizados son la sensibilidad, especificidad, valor predictivo positivo y valor predictivo negativo. Por lo tanto una EVRUPP ideal es aquella que tenga buen valor predictivo (tanto positivo como negativo), alta sensibilidad y especificidad, facilidad de usar y que presente criterios claros y definidos que eviten al máximo la variabilidad entre observadores ${ }^{5}$.

Los pacientes críticos que se encuentran en Unidades de Cuidado Intensivo, por las patologías que sufren y la situación aguda que presentan, están expuestos a más intensos y variados factores de riesgo tales como fármacos vaso-activos, relajantes musculares, disminución del nivel de conciencia, entre otros, que los hacen propensos a desarrollar las úlceras 
escala específica que calcule de manera válida, fiable y rápida los riesgos de presentar estas lesiones.

El uso de las escalas de valoración tiene cinco ventajas para las Instituciones de Salud donde se aplican, que son asegurar la asignación eficiente y efectiva de recursos preventivos limitados, servir de soporte en las decisiones clínicas, permitir el ajuste de casos en función del riesgo en estudios epidemiológicos, facilitar el desarrollo de protocolos de valoración del riesgo y servir como prueba en casos de litigios ${ }^{6}$.

Sin embargo las ventajas más importantes que tiene el uso de estas escalas en la Institución de Salud, son disminuir el impacto financiero que genera el tratamiento de estas lesiones y garantizar la Seguridad del Paciente.

El objetivo de este estudio fue determinar la sensibilidad, especificidad, valor predictivo positivo de la escala de Braden y la escala de EVARUCI, al ser aplicadas en una Unidad de Cuidados Intensivos Adulto de una Institución Prestadora de Servicios de Salud nivel IV en Cali en el año 2012.

\section{Materiales y Metodos}

La investigación se baso en un estudio de validez de tipo analítico, observacional, longitudinal, prospectivo y de cohortes $^{7}$, con dos EVRUPP, realizado en una unidad de cuidados intensivos, de una institución de IV nivel de atención de la ciudad de Cali (Colombia).

Se seleccionaron consecutivamente pacientes mayores de edad, sin ulceras por presión a su ingreso a la UCI, que accedieron a participar del estudio mediante la firma del consentimiento informado, si el paciente presentaba limitación de tipo sensorial o cognitivo, la firma del consentimiento la efectuó un familiar hasta tercer grado de consanguinidad. Se aplicaron ambas escalas de 0 a 7 días consecutivamente, posteriormente cada 3 días hasta completar 7 semanas tiempo de terminación del estudio, en el periodo de tiempo comprendido entre febrero y abril de 2012 .

La variable dependiente del estudio fue la Ulcera por Presión se asociaron como covariables la edad, el sexo y el peso corporal, y como variables de exposición, los días de estancia en la Unidad de Cuidados Intensivos, el diagnostico, los antecedentes patológicos, la movilidad, la actividad, la nutrición, exposición a la humedad, percepción sensorial, fricción, estado de conciencia, estado hemodinámico, estado respiratorio, temperatura corporal, saturación de oxígeno, presión arterial, estado de la piel y posición del paciente, ya que son considerados factores causales del problema; también se tuvieron en cuenta los puntajes de las escalas aplicadas (Braden y EVARUCI).

\section{Resultados}

La muestra consistió en un total 355 pacientes $50.7 \%$ de género masculino y $49.3 \%$ de género femenino, Las edades oscilaron entre 18 y 92 años, con un promedio de 58.55 años, el promedio de estancia hospitalaria fue de 5.2 días, el $50 \%$ de los pacientes tuvieron una estancia menor o igual a 4 días. El diagnostico de base más frecuente fue de origen cardiovascular $(24,2 \%)$ seguido del de origen infeccioso (10,7\%). El 85\% de los pacientes (302 personas) terminaron el estudio por presentar egreso hospitalario de la Unidad de Cuidados Intensivos, el $11 \%$ (38 personas) por haber desarrollado úlcera por presión y el 4\% (15 personas) por haber fallecido. La localización más frecuente de la úlcera por presión fue en los glúteos (61\%), seguida del sacro (24\%), de los talones (11\%), de los trocánteres $(3 \%)$ y de los codos $(3 \%)$.

A partir de los resultados obtenidos, se comparó el comportamiento de ambas escalas, teniendo en cuenta los criterios de validez, en el inicio, mitad y final de la hospitalización.

Tabla 1. Comparación Sensibilidad Escala de Braden y Escala de EVARUCI

\begin{tabular}{c|cccc}
\hline Evaluación & \multicolumn{1}{c}{ Braden } & \multicolumn{1}{c}{ EVARUCI } & \multicolumn{1}{c}{ Significancia } \\
\hline Inicio & $76.3 \%$ & $55.3 \%$ & 0.05 \\
\hline Medio & $65.8 \%$ & $57.9 \%$ & 0.48 \\
\hline Final & $65.8 \%$ & $57.9 \%$ & 0.48 \\
\hline
\end{tabular}

En las tres evaluaciones, se obtuvo que la sensibilidad de la escala de Braden en la evaluación inicial, media y final es mayor a la de la escala de EVARUCI, pero solo en la evaluación inicial existe una verdadera diferencia, con una significancia de 0.05

Tabla 2. Comparación Especificidad Escala de Braden y Escala de EVARUCI

\begin{tabular}{c|rrrr}
\hline Evaluación & \multicolumn{1}{c}{ Braden } & \multicolumn{1}{c}{ EVARUCI } & Significancia \\
\hline Inicio & $77.6 \%$ & $84.2 \%$ & 0.03 \\
\hline Medio & $81,7 \%$ & $88.3 \%$ & 0.02 \\
\hline Final & $86.1 \%$ & $94 \%$ & 0.00 \\
\hline
\end{tabular}

En las tres evaluaciones, se observó que la especificidad de de la escala de EVARUCI en la evaluación inicial, media y final es mayor a la de la escala de Braden, con una significancia de $0.03,0.02$ y 0.00 respectivamente.

Tabla 3. Comparación Valor Predictivo Positivo Escala de Braden y Escala de EVARUCI

\begin{tabular}{|cccc}
\hline Evaluación & \multicolumn{1}{c}{ Braden } & \multicolumn{1}{c}{ EVARUCI } & Significancia \\
\hline Inicio & $29 \%$ & $29.6 \%$ & 0.93 \\
\hline Medio & $30.1 \%$ & $37.3 \%$ & 0.33 \\
\hline Final & $36.2 \%$ & $53.7 \%$ & 0.04 \\
\hline
\end{tabular}

$\mathrm{Al}$ observar la tabla 3 se pudo inferir que el valor predictivo positivo de la escala de EVARUCI es mayor al de la escala de Braden en las tres evaluaciones realizadas, pero solo al final de la hospitalización se registra una diferencia significativa de 0.04 . 
escala específica que calcule de manera válida, fiable y rápida los riesgos de presentar estas lesiones.

El uso de las escalas de valoración tiene cinco ventajas para las Instituciones de Salud donde se aplican, que son asegurar la asignación eficiente y efectiva de recursos preventivos limitados, servir de soporte en las decisiones clínicas, permitir el ajuste de casos en función del riesgo en estudios epidemiológicos, facilitar el desarrollo de protocolos de valoración del riesgo y servir como prueba en casos de litigios ${ }^{6}$.

Sin embargo las ventajas más importantes que tiene el uso de estas escalas en la Institución de Salud, son disminuir el impacto financiero que genera el tratamiento de estas lesiones y garantizar la Seguridad del Paciente.

El objetivo de este estudio fue determinar la sensibilidad, especificidad, valor predictivo positivo de la escala de Braden y la escala de EVARUCI, al ser aplicadas en una Unidad de Cuidados Intensivos Adulto de una Institución Prestadora de Servicios de Salud nivel IV en Cali en el año 2012.

\section{Materiales y Metodos}

La investigación se baso en un estudio de validez de tipo analítico, observacional, longitudinal, prospectivo y de cohortes $^{7}$, con dos EVRUPP, realizado en una unidad de cuidados intensivos, de una institución de IV nivel de atención de la ciudad de Cali (Colombia).

Se seleccionaron consecutivamente pacientes mayores de edad, sin ulceras por presión a su ingreso a la UCI, que accedieron a participar del estudio mediante la firma del consentimiento informado, si el paciente presentaba limitación de tipo sensorial o cognitivo, la firma del consentimiento la efectuó un familiar hasta tercer grado de consanguinidad. Se aplicaron ambas escalas de 0 a 7 días consecutivamente, posteriormente cada 3 días hasta completar 7 semanas tiempo de terminación del estudio, en el periodo de tiempo comprendido entre febrero y abril de 2012 .

La variable dependiente del estudio fue la Ulcera por Presión se asociaron como covariables la edad, el sexo y el peso corporal, y como variables de exposición, los días de estancia en la Unidad de Cuidados Intensivos, el diagnostico, los antecedentes patológicos, la movilidad, la actividad, la nutrición, exposición a la humedad, percepción sensorial, fricción, estado de conciencia, estado hemodinámico, estado respiratorio, temperatura corporal, saturación de oxígeno, presión arterial, estado de la piel y posición del paciente, ya que son considerados factores causales del problema; también se tuvieron en cuenta los puntajes de las escalas aplicadas (Braden y EVARUCI).

\section{Resultados}

La muestra consistió en un total 355 pacientes $50.7 \%$ de género masculino y $49.3 \%$ de género femenino, Las edades oscilaron entre 18 y 92 años, con un promedio de 58.55 años, el promedio de estancia hospitalaria fue de 5.2 días, el $50 \%$ de los pacientes tuvieron una estancia menor o igual a 4 días. El diagnostico de base más frecuente fue de origen cardiovascular $(24,2 \%)$ seguido del de origen infeccioso $(10,7 \%)$. El $85 \%$ de los pacientes (302 personas) terminaron el estudio por presentar egreso hospitalario de la Unidad de Cuidados Intensivos, el $11 \%$ (38 personas) por haber desarrollado úlcera por presión y el $4 \%$ (15 personas) por haber fallecido. La localización más frecuente de la úlcera por presión fue en los glúteos (61\%), seguida del sacro (24\%), de los talones (11\%), de los trocánteres (3\%) y de los codos (3\%).

A partir de los resultados obtenidos, se comparó el comportamiento de ambas escalas, teniendo en cuenta los criterios de validez, en el inicio, mitad y final de la hospitalización.

Tabla 1. Comparación Sensibilidad Escala de Braden y Escala de EVARUCI

\begin{tabular}{|c|c|c|c|}
\hline Evaluación & \multicolumn{1}{c}{ Braden } & \multicolumn{1}{c}{ EVARUCI } & Significancia \\
\hline \multicolumn{1}{c}{ Inicio } & $76.3 \%$ & $55.3 \%$ & 0.05 \\
\hline Medio & $65.8 \%$ & $57.9 \%$ & 0.48 \\
\hline Final & $65.8 \%$ & $57.9 \%$ & 0.48 \\
\hline
\end{tabular}

En las tres evaluaciones, se obtuvo que la sensibilidad de la escala de Braden en la evaluación inicial, media y final es mayor a la de la escala de EVARUCI, pero solo en la evaluación inicial existe una verdadera diferencia, con una significancia de 0.05

Tabla 2. Comparación Especificidad Escala de Braden y Escala de EVARUCI

\begin{tabular}{|c|r|r|c|}
\hline Evaluación & \multicolumn{1}{l}{ Braden } & \multicolumn{1}{l}{ EVARUCI } & Significancia \\
\hline Inicio & $77.6 \%$ & $84.2 \%$ & 0.03 \\
\hline Medio & $81,7 \%$ & $88.3 \%$ & 0.02 \\
\hline Final & $86.1 \%$ & $94 \%$ & 0.00 \\
\hline
\end{tabular}

En las tres evaluaciones, se observó que la especificidad de de la escala de EVARUCI en la evaluación inicial, media y final es mayor a la de la escala de Braden, con una significancia de $0.03,0.02$ y 0.00 respectivamente.

Tabla 3. Comparación Valor Predictivo Positivo Escala de Braden y Escala de EVARUCI

\begin{tabular}{|c|c|c|c|}
\hline Evaluación & \multicolumn{1}{c}{ Braden } & EVARUCI & Significancia \\
\hline Inicio & $29 \%$ & $29.6 \%$ & 0.93 \\
\hline Medio & $30.1 \%$ & $37.3 \%$ & 0.33 \\
\hline Final & $36.2 \%$ & $53.7 \%$ & 0.04 \\
\hline
\end{tabular}

Al observar la tabla 3 se pudo inferir que el valor predictivo positivo de la escala de EVARUCI es mayor al de la escala de Braden en las tres evaluaciones realizadas, pero solo al final de la hospitalización se registra una diferencia significativa de 0.04 . 
escala específica que calcule de manera válida, fiable y rápida los riesgos de presentar estas lesiones.

El uso de las escalas de valoración tiene cinco ventajas para las Instituciones de Salud donde se aplican, que son asegurar la asignación eficiente y efectiva de recursos preventivos limitados, servir de soporte en las decisiones clínicas, permitir el ajuste de casos en función del riesgo en estudios epidemiológicos, facilitar el desarrollo de protocolos de valoración del riesgo y servir como prueba en casos de litigios ${ }^{6}$.

Sin embargo las ventajas más importantes que tiene el uso de estas escalas en la Institución de Salud, son disminuir el impacto financiero que genera el tratamiento de estas lesiones y garantizar la Seguridad del Paciente.

El objetivo de este estudio fue determinar la sensibilidad, especificidad, valor predictivo positivo de la escala de Braden y la escala de EVARUCI, al ser aplicadas en una Unidad de Cuidados Intensivos Adulto de una Institución Prestadora de Servicios de Salud nivel IV en Cali en el año 2012.

\section{Materiales y Metodos}

La investigación se baso en un estudio de validez de tipo analítico, observacional, longitudinal, prospectivo y de cohortes $^{7}$, con dos EVRUPP, realizado en una unidad de cuidados intensivos, de una institución de IV nivel de atención de la ciudad de Cali (Colombia).

Se seleccionaron consecutivamente pacientes mayores de edad, sin ulceras por presión a su ingreso a la UCI, que accedieron a participar del estudio mediante la firma del consentimiento informado, si el paciente presentaba limitación de tipo sensorial o cognitivo, la firma del consentimiento la efectuó un familiar hasta tercer grado de consanguinidad. Se aplicaron ambas escalas de 0 a 7 días consecutivamente, posteriormente cada 3 días hasta completar 7 semanas tiempo de terminación del estudio, en el periodo de tiempo comprendido entre febrero y abril de 2012 .

La variable dependiente del estudio fue la Ulcera por Presión se asociaron como covariables la edad, el sexo y el peso corporal, y como variables de exposición, los días de estancia en la Unidad de Cuidados Intensivos, el diagnostico, los antecedentes patológicos, la movilidad, la actividad, la nutrición, exposición a la humedad, percepción sensorial, fricción, estado de conciencia, estado hemodinámico, estado respiratorio, temperatura corporal, saturación de oxígeno, presión arterial, estado de la piel y posición del paciente, ya que son considerados factores causales del problema; también se tuvieron en cuenta los puntajes de las escalas aplicadas (Braden y EVARUCI).

\section{Resultados}

La muestra consistió en un total 355 pacientes $50.7 \%$ de género masculino y $49.3 \%$ de género femenino, Las edades oscilaron entre 18 y 92 años, con un promedio de 58.55 años, el promedio de estancia hospitalaria fue de 5.2 días, el 50\% de los pacientes tuvieron una estancia menor o igual a 4 días. El diagnostico de base más frecuente fue de origen cardiovascular $(24,2 \%)$ seguido del de origen infeccioso $(10,7 \%)$. El $85 \%$ de los pacientes (302 personas) terminaron el estudio por presentar egreso hospitalario de la Unidad de Cuidados Intensivos, el $11 \%$ (38 personas) por haber desarrollado úlcera por presión y el $4 \%$ (15 personas) por haber fallecido. La localización más frecuente de la úlcera por presión fue en los glúteos (61\%), seguida del sacro (24\%), de los talones (11\%), de los trocánteres $(3 \%)$ y de los codos $(3 \%)$.

A partir de los resultados obtenidos, se comparó el comportamiento de ambas escalas, teniendo en cuenta los criterios de validez, en el inicio, mitad y final de la hospitalización.

Tabla 1. Comparación Sensibilidad Escala de Braden y Escala de EVARUCI

\begin{tabular}{|c|c|c|c|}
\hline Evaluación & \multicolumn{1}{c}{ Braden } & EVARUCI & Significancia \\
\hline \multicolumn{1}{c}{ Inicio } & $76.3 \%$ & $55.3 \%$ & 0.05 \\
\hline Medio & $65.8 \%$ & $57.9 \%$ & 0.48 \\
\hline Final & $65.8 \%$ & $57.9 \%$ & 0.48 \\
\hline
\end{tabular}

En las tres evaluaciones, se obtuvo que la sensibilidad de la escala de Braden en la evaluación inicial, media y final es mayor a la de la escala de EVARUCI, pero solo en la evaluación inicial existe una verdadera diferencia, con una significancia de 0.05

Tabla 2. Comparación Especificidad Escala de Braden y Escala de EVARUCI

\begin{tabular}{|c|r|r|c|}
\hline Evaluación & \multicolumn{1}{l}{ Braden } & EVARUCI & Significancia \\
\hline Inicio & $77.6 \%$ & $84.2 \%$ & 0.03 \\
\hline Medio & $81,7 \%$ & $88.3 \%$ & 0.02 \\
\hline Final & $86.1 \%$ & $94 \%$ & 0.00 \\
\hline
\end{tabular}

En las tres evaluaciones, se observó que la especificidad de de la escala de EVARUCI en la evaluación inicial, media y final es mayor a la de la escala de Braden, con una significancia de $0.03,0.02$ y 0.00 respectivamente.

Tabla 3. Comparación Valor Predictivo Positivo Escala de Braden y Escala de EVARUCI

\begin{tabular}{ccccc}
\hline Evaluación & \multicolumn{1}{c}{ Braden } & EVARUCI & Significancia \\
\hline Inicio & $29 \%$ & $29.6 \%$ & 0.93 \\
\hline Medio & $30.1 \%$ & $37.3 \%$ & 0.33 \\
\hline Final & $36.2 \%$ & $53.7 \%$ & 0.04 \\
\hline
\end{tabular}

$\mathrm{Al}$ observar la tabla 3 se pudo inferir que el valor predictivo positivo de la escala de EVARUCI es mayor al de la escala de Braden en las tres evaluaciones realizadas, pero solo al final de la hospitalización se registra una diferencia significativa de 0.04 . 
Tabla 4. Comparación Valor Predictivo Negativo Escala de Braden y Escala de EVARUCI

\begin{tabular}{c|cccc}
\hline \multicolumn{1}{c}{ Evaluación } & \multicolumn{1}{c}{ Braden } & \multicolumn{1}{c}{ EVARUCI } & \multicolumn{1}{c}{ Significancia } \\
\hline \multicolumn{1}{c}{ Inicio } & $96.5 \%$ & $94 \%$ & 0.20 \\
\hline Medio & $95.2 \%$ & $95.6 \%$ & 0.74 \\
\hline Final & $95.5 \%$ & $94.9 \%$ & 0.76 \\
\hline
\end{tabular}

Al observar la tabla 4 se obtuvo que el valor predictivo negativo en la evaluación inicial es superior en la escala de Braden, en la evaluación media es superior en la escala de EVARUCI y en la evaluación final es mayor en la escala de Braden, pero en ninguno de los tres casos hay diferencias significativas.

\section{Grafico 1. Sencibilidad Escala de Braden y Escala de} EVARUCI

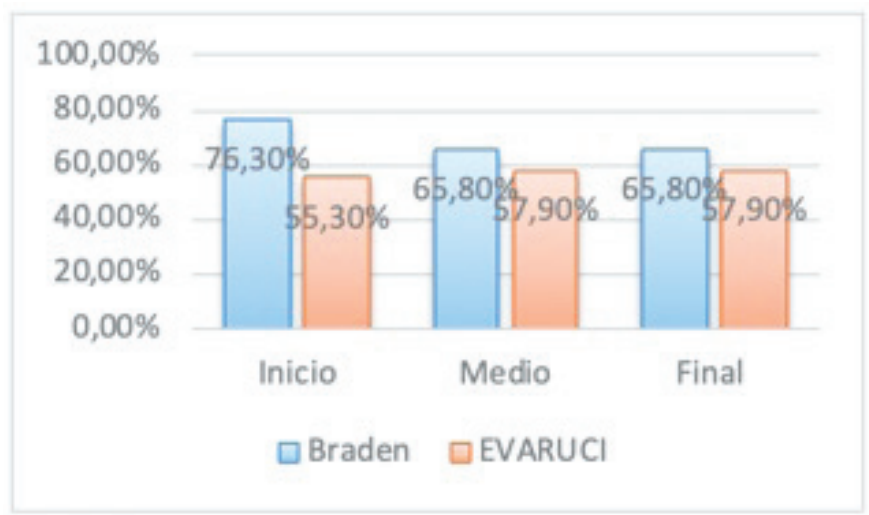

La grafica anterior muestra la distribución del porcentaje estimado de la escala Braden y Escala de EVARUCI para evaluar sensibilidad en la aparición de ulceras, se puede observar que las dos escalas se comportan muy similarmente con porcentajes superiores al 50\%, aunque la escala Braden presento mejores resultados en cuanto a la presencia de ulcera, solo se presentaron diferencias estadísticas significativas entre las escalas en la fase de inicio $(\mathrm{p}<0,05)$.

\section{Grafico 1. Especificidad Escala de Braden y Escala de} EVARUC

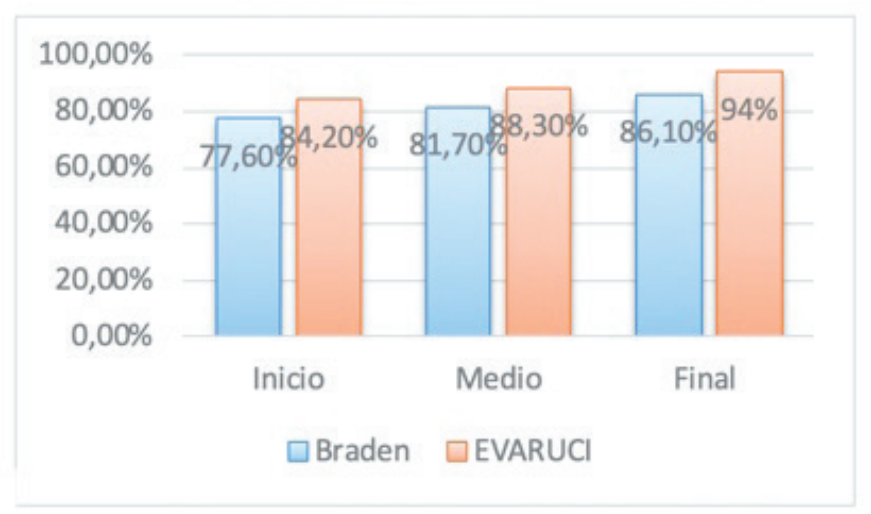

La grafica anterior muestra la distribución del porcentaje estimado de la escala Braden y Escala de EVARUCI para evaluar especificidad en la aparición de ulceras, se puede observar que las dos escalas se comportan muy similarmente con porcentajes superiores al $70 \%$, aunque la escala EVARUCI presento mejores resultados en cuanto a la identificación de los pacientes libres de ulcera, en las tres fases del análisis se presentaron diferencias estadísticas significativas $(\mathrm{p}<0,05)$.

Grafico 3. Comparación Valor Predictivo Positivo Escala de Braden y Escala de EVARUCI

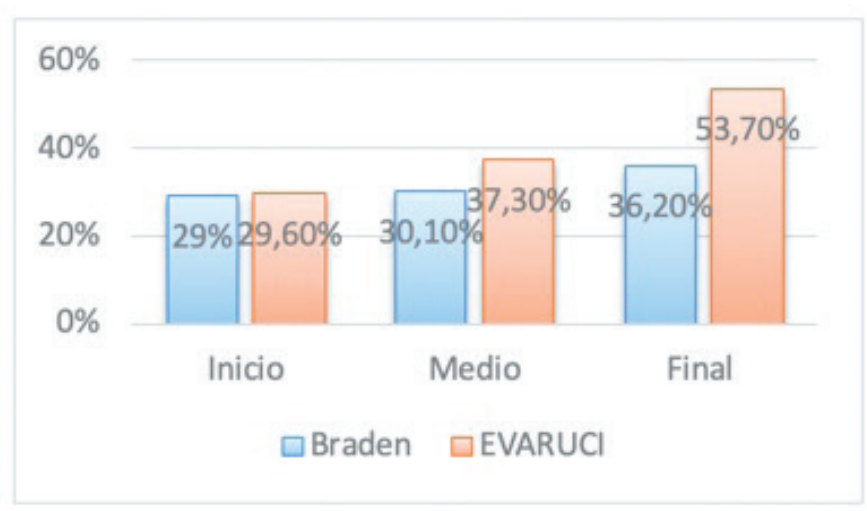

La grafica anterior muestra el valor predictivo positivo de la escala de la escala Barden y EVARUCI, se puede observar que la escala Evaruci presento mejores resultados en las tres evaluaciones realizadas, aunque los valores fueron inferiores al 50\%, el mejor pronóstico fue al final de la hospitalización donde se encontraron diferencias estadísticas significativa $(\mathrm{p}<0.05)$.

\section{Grafico 4. Comparación Valor Predictivo Negativo Escala de Braden y Escala de EVARUCI}

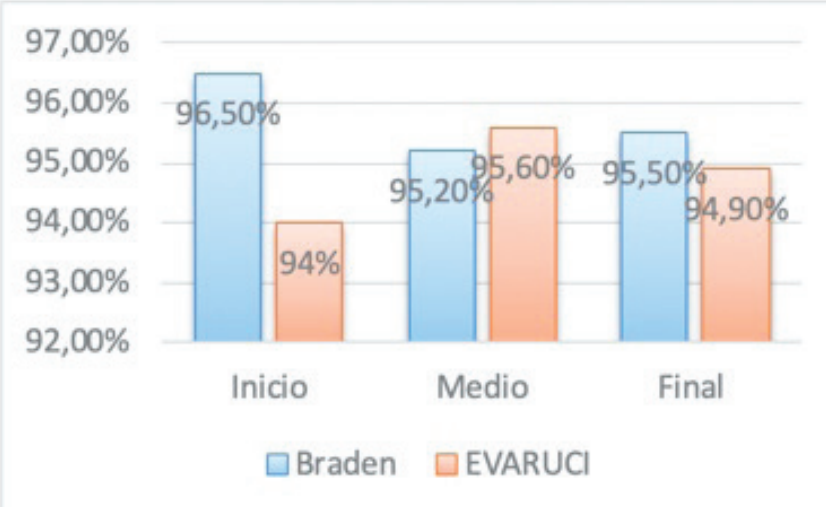

La grafica anterior se puede observar los resultados obtenidos en el valor predictivo negativo, en la evaluación inicial y final los valores de la escala de Braden fueron superiores a la Evaruci, y en la evaluación media los resultados fueron contrarios, la escala de EVARUCI fue superior a la Braden, no se encontraron diferencias estadisitcas significativas en ninguno de los casos $(\mathrm{p}>0,05)$. 


\section{Discusion}

Las úlceras por presión (UPP) son lesiones de etiología única y conocida, algunos autores muestran que estas están relacionadas con múltiples factores de riesgo. Los pacientes críticos, por las patologías que sufren y la situación aguda que presentan, están expuestos a más intensos y variados factores de riesgo ${ }^{(8-10)}$. Por ello, la aparición de UPP es un problema recurrente en pacientes institucionalizados y mucho más frecuente en prevalencia e incidencia en los pacientes ingresados en Unidades de Cuidados Intensivos (UCI).

Es claro que los procesos de intervención deben ser valorados y estudiados para evitar procesos invasivos innecesarios, sobre todo cuando estos producen efectos sobre la salud dado el caso. Contar con un instrumento predictivo de riesgo de desarrollar úlceras, puede ser considerado de gran utilidad para poder realizar una mejor prevención. ${ }^{(11)}$ Los resultados de Blumel J. (12), demuestran que, en nuestro medio, la escala de Braden es un instrumento capaz de predecir el riesgo de desarrollar úlceras por presión con una adecuada sensibilidad y especificidad. Sin embargo, los resultados de otros trabajos muestran un amplio rango de sensibilidades (entre 35-100\%) debido, probablemente, a que fueron realizados en distintos ambientes y con poblaciones diferentes, por ejemplo, en pacientes hospitalizados, ancianos en casas de reposo, en atención primaria, entre otros, ${ }^{(13-16)}$ al comparar con este estudio los resultados de las predicción fueron similares en sensibilidad (65\% a 76\%), en especificidad ( $77 \%$ a $86 \%)$.

Para el caso de Evaruci, el estudio de González J.M. (9), las puntuaciones iniciales y finales fueron: sensibilidad $(100 \%$, $100 \%, 90,91 \%)$, especificidad $(68,63 \%, 49,02 \%, 92,16 \%)$, valor predictivo positivo $(40,74 \%, 29,73 \%, 71,43 \%)$, valor predictivo negativo $(100 \%, 100 \%, 97,2 \%)$, a pesar que el estudio de González mostro buenos resultados para Evaruci al compararlo con este estudio sensibilidad en las puntuaciones iniciales medias y finales fueron inferiores, $(55,30 \%, 57,90 \%$ y $57,90 \%)$, para la especificidad fueron superiores $(84,20 \%$, $88,30 \%$ y $94 \%)$ en cuanto a valor predictivo positivo $(29,60 \%$, $37,30 \%$ y $53,70 \%$ ) se tuvieron resultados similares, y por último el valor predictivo negativo $(94 \%, 95,60 \%$ y $94,90 \%)$ se obtuvieron resultados similares.

$\mathrm{Al}$ observar el grafico se pudo inferir que la de la escala de EVARUCI en las tres evaluaciones es más alta que la de la escala de Braden, lo cual indica que esta última escala es más específica, es decir, tiene mayor probabilidad de clasificar correctamente a un paciente sin úlcera por presión, además, tiene un resultado del valor predictivo negativo no fueron significativos para la escala. El nivel de significancia en las tres evaluaciones es alto, lo que quiere decir que los valores son concluyentes. Estos resultados difieren con el de las evaluaciones iniciales y media de los estudios de validez de la escalas, en los cuales los porcentajes de la escala de EVARUCI son menores que los de la escala de Braden, pero coinciden con los resultados finales, en los cuales el porcentaje de especificidad de la escala de EVARUCI es mayor que el de la escala de Braden. Esta diferencia pudo obedecer al hecho de que a medida de que va pasando el tiempo la especificidad de las escalas aumenta y mucho más la de la escala de EVARUCI, ya que una vez identificado el riesgo, se van realizando medidas para evitar que las úlceras aparezcan, lo cual hace que al final el test tenga la capacidad adecuada para identificar a los pacientes sin úlceras, es decir a los verdaderos negativos.

Al observar los porcentajes de los valores predictivos positivos de ambas escalas, se obtuvo que en las tres evaluaciones los valores de la escala de Braden son menores que los de la escala de EVARUCI, indicando así, que esta última escala tiene un valor predictivo positivo más alto, lo cual significa que cuando un paciente tiene un resultado positivo en la prueba puede llegar a desarrollar una úlcera por presión. Sin embargo estos resultados cobran importancia en la evaluación final con una significancia de 0.04 .

Al observar los porcentajes de los valores predictivos negativos de ambas escalas, se concluye que el valor obtenido en la evaluación inicial y final en la escala de Braden son mayores que los de la escala de EVARUCI, pero el valor obtenido en la evaluación media de la escala de Braden es menor, además el nivel de significancia en las tres evaluaciones no es concluyente. Lo cual significa que ambas escalas son capaces de predecir que un paciente estará sano, es decir no desarrollara una ulcera por presión cuando tenga un resultado negativo en las pruebas. Estos resultados difieren un poco con los obtenidos en los estudios de validez de las escalas, ya que en el de la escala de EVARUCI los resultados fueron más altos en las tres evaluaciones, y el porcentaje del valor predictivo negativo de ambas escala disminuyo en cada evaluación, mientras que en este solo disminuyo el de Braden, pero el de EVARUCI aumento. Esta diferencia puede estar relacionada con los mismos hechos que explican la variabilidad en la sensibilidad, con relación al número de pacientes, ya que la escala de EVARUCI parece que con el aumento de la estancia hospitalaria también capta mejor los verdaderos negativos.

\section{Conclusiones}

Todos los criterios de validez de una prueba diagnóstica o de un test son importantes, ya que cada uno toma un aspecto distinto de la situación de un paciente, ya sea en el instante o en el futuro, sin embargo, en el caso de las escalas de valoración del riesgo de presentar desarrollar úlceras por presión, la validez radica en los valores predictivos, ya que el objetivo de la escala es identificar de forma precoz a los pacientes que pueden presentar las lesiones en la piel, con base en la presencia de factores de riesgo, para consecuentemente aplicar unas medidas preventivas en función del nivel de riesgo y evitar que la úlcera aparezca.

Desde este punto de vista la escala de EVARUCI es una buena herramienta en la prevención de úlceras por presión, ya que tiene un valor predictivo positivo un poco más alto que la escala de Braden. El valor predictivo positivo de ambas escalas inicia siendo muy bajo, pero va aumentando progresivamente a medida que trascurre la estancia hospitalaria, sin embargo, el porcentaje de la escala de EVARUCI se ubica todo el tiempo por encima del de la escala de Braden, notándose aún más en la última evaluación, es decir antes de que el paciente desarrolle la lesión o salga del estudio. 
El valor predictivo negativo de ambas escalas fue similar en la segunda y tercera evaluación, con porcentajes relativamente altos, lo cual indica que ambas escalas detectan bastante bien los verdaderos negativos.

Teniendo en cuenta estos dos aspectos, se pudo concluir que la escala de EVARUCI es un instrumento predictor valido para la detección del riesgo de desarrollar úlceras por presión en pacientes críticos y que además valora aspectos propios de las Unidades de Cuidados Intensivos, tales como el nivel de consciencia, el estado hemodinámico, el soporte de oxígeno, la movilidad, la temperatura, la saturación, la presión arterial, el estado de la piel y la posición del paciente, que en caso de estar alterados representan un riesgo de desarrollar úlceras por presión, ya que el único factor que influye en la aparición de estas lesiones, no es la posición del paciente, sino condiciones propias de hipoperfusión en la piel.

\section{Referencias}

1. European Pressure Ulcer Advisory Panel and National Pressure Ulcer Advisory Panel. Prevention and treatment of pressure ulcers: quick reference guide. Washington DC: National Pressure Ulcer Advisory Panel; 2009. P. 5 Disponible en: http://www.epuap.org/guidelines/QRG Prevention in Spanish.pdf

2. Águila Pollo M.C., Corrales Pérez J.M., Buitrago Barrasa V., Serrano Monge V., Ramos Sánchez A., Fernández Ribeiro A.S. Prevalencia de úlceras por presión en un centro sociosanitario. Ene. [revista en la Internet]. 2014 [citado 2015 Sep 26] ; 8(2): Disponible en: http:// scielo.isciii.es/scielo.php?script=sci arttext\&pid=S1988348X2014000200007\&lng=es

3. Ramos A., Ribeiro A.S., Martín A., Vázquez M., Blanco B., Corrales J.M. et al. Prevalencia de úlceras por presión en un centro sociosanitario de media-larga estancia. Gerokomos [revista en la Internet]. 2013 Mar [citado 2015 Sep 26]; 24(1): 36-40. Disponible en: http://scielo. isciii.es/ scielo.php?script $=$ sci_arttext\&pid $=\mathrm{S} 1134$ 928X2013000100008\&lng=es

4. Espinosa Manríquez E. Ulceras por presión. 2012. P. 1 Disponible en: http://www.facmed.unam.mx/ deptos/salud/censenanza/spivsa/antol\%202\%20 anciano/2parte2013/ulceras.pdf

5. Ballabriga M. Validación de la escala de Norton en un Hospital de Geriatria (Análisis preliminar de la escala EMINA como herramienta alternativa). 2a edición. Edita Colegio Oficial de Enfermeria Huesca. 2007. P. 4

6. García Fernández F.P., Pancorbo Hidalgo P.L., Soldevilla Agreda J.J. Escalas de valoración del riesgo de desarrollar úlceras por presión en la infancia. Gerokomos [revista en la Internet]. 2011 Mar [citado 2015 Sep 26]; 22(1): 26-34. Disponible en: http://scielo. isciii.es/scielo.php? script $=$ sci $\quad$ arttext\&pid $=\mathrm{S} 1134-$ 928X2011000100005\&lng $=\mathrm{es}$

7. Argimon Pallas J.M., Jiménez Villa J. Métodos de
Investigación Clínica y Epidemiológica. $3^{\mathrm{a}}$ Edición. Madrid. Elsevier. 2004 P. 28-32.

8. Cubbin B, Jackson C. Trial of a pressure area risk calculator for intensive therapy patients. Intensive Care Nurs. 1991;7:40-4.

9. González Ruiz JM, González Carrero AA, Heredero Blázquez MT, de Vera Vera R, González Ortiz B, Pulido $\mathrm{M}$, et al. Factores de riesgo de úlceras por presión en pacientes críticos. Enferm Clin. 2001;3:97-103.

10. Batson S, Adam S, Hall G, Quirke S. The development of a 129 pressure area scoring system for critically ill patients: a pilot estudy. Intensive Crit Care Nurs. 1993;9:146-51.

11. Mazzocco R, Zampieron A. Does the evaluation of the pressure ulcer risk increase better prevention? Prof Inferm 2000; 53: 173-8.

12. Blümel M Juan E, Tirado Ga Karina, Schiele Ma Claudia, Schönffeldt Fa Gabriela, Sarrá C Salvador. Validez de la escala de Braden para predecir úlceras por presión en población femenina. Rev Méd Chile 2004; 132: 595-600.

13. Boes C. Reliability and validity of the Braden Scale for predicting pressure sore risk. Pflege. 2000; 13: 397-402.

14. Pang S, Wong T. Predicting pressure sore risk with the Norton, Braden and Waterlow scales in a Hong Kong rehabilitation hospital. Nursing Research 1998; 47: 14753. 11 .

15. Ramundo JM. Reliability and validity of the Braden Scale in the home care setting. J Wound Ostomy Continence Nurs 1995; 22: 128-34. 12.

16. Barnes D, Payton RG. Clinical application of the Braden Scale in the acute care. Dermatol Nurs 1993; 5: 386-8. 